Pacific Journal of Mathematics

ON MEANS OF DISTANCES ON THE SURFACE OF A SPHERE. 


\section{ON MEANS OF DISTANCES \\ ON THE SURFACE OF A SPHERE. II \\ (UPPER BOUNDS)}

\section{Gerold WAGNeR ${ }^{1}$}

Given $N$ points $x_{1}, \ldots, x_{N}$ on the unit sphere $S$ in Euclidean $d$ space $(d \geq 3)$, lower bounds for the deviation of the sum $\sum\left|x-x_{j}\right|^{\alpha}$, $\alpha>1-d ; x \in S$, from its mean value were established in terms of $L^{1}$-norms in the first part of this paper. In the present part it is shown that these bounds are best possible. Our main tool is a multidimensional quadrature formula with equal weights.

1. Introduction. On the surface $S=S^{d-1}$ of the unit sphere in $d$ dimensional Euclidean space $E^{d} \quad(d \geq 3)$, we consider a certain class of distance functions and distance functionals, associated with a given $N$ point set $\omega_{N}=\left\{x_{1}, x_{2}, \ldots, x_{N}\right\}$ on $S$. Denote by $|x-y|$ the Euclidean distance between two points $x$ and $y$ in $E^{d}$. Let $x \in S^{d-1}$ be a variable point. For each value of a parameter $\alpha(1-d<\alpha<\infty)$ consider the distance function $U_{\alpha}\left(x, \omega_{N}\right)$ which we define as follows:

$$
U_{\alpha}\left(x, \omega_{N}\right)=\sum_{j=1}^{N}\left|x-x_{j}\right|^{\alpha}-N \cdot m(\alpha, d) \quad \text { for } \alpha \neq 0,
$$

and

$$
U_{0}\left(x, \omega_{N}\right)=\sum_{j=1}^{N} \log \left|x-x_{j}\right|-N \cdot m(0, d) .
$$

Here $m(\alpha, d)$ denotes the mean value of $\left|x-x_{j}\right|^{\alpha}$ on $S^{d-1}$, i.e.

$$
\begin{aligned}
& m(\alpha, d)=\frac{1}{\sigma(S)} \int_{S}\left|x-x_{j}\right|^{\alpha} d \sigma(x) \quad \text { for } \alpha \neq 0, \\
& m(0, d)=\frac{1}{\sigma(S)} \int_{S} \log \left|x-x_{j}\right| d \sigma(x),
\end{aligned}
$$

where $\sigma$ is the $(d-1)$-dimensional area measure on $S^{d-1}$.

In the first part [4] we proved certain lower bounds for the $L^{1}$-norms of the functions $U_{\alpha}\left(x, \omega_{N}\right)$ (see Theorem 1 in [4]). The existence of such lower bounds is due to the fact that uniform distribution on $S^{d-1}$

\footnotetext{
${ }^{1}$ The author died on March 10, 1990 in a skiing accident in Austria.
} 
can be approximated by an $N$ point distribution to a certain degree of accuracy only. In this part we will show that the lower bounds obtained in [4] are best possible, apart from the values of certain constants. More precisely, we shall prove:

THEOREM A. For any $\alpha>1-d$ and some positive constant $c=$ $c(\alpha, d)$, there exists, for each $N \geq 1$, an $N$-tuple $\omega_{N}^{0}$ of points $S^{d-1}$ (depending on $\alpha$ ) such that the following relations hold:

(a) $\max _{x \in S}\left|U_{\alpha}\left(x, \omega_{N}^{0}\right)\right| \leq c(\alpha, d) \cdot N^{-\alpha /(d-1)}$ if $0<\alpha<\infty ; \alpha \neq$ $-2,4, \ldots$,

(b) $\min _{x \in S} U_{\alpha}\left(x, \omega_{N}^{0}\right) \leq c(\alpha, d) \cdot N^{-\alpha /(d-1)}$ if $1-d<\alpha<0$,

(c) $\max _{x \in S} U_{0}\left(x, \omega_{N}^{0}\right) \leq(0, d)$ if $\alpha=0$,

(d) $U_{\alpha}\left(x, \omega_{N}^{0}\right) \equiv 0$ if $\alpha \in\{2,4, \ldots\}$ and $N>N_{0}(\alpha, d)$.

In view of the relations $\int_{S} U_{\alpha}\left(x, \omega_{N}\right) d(\sigma(x))=0$, the bounds in (a)-(c) are also upper bounds for the $L^{1}$-norms

$$
\frac{1}{\sigma(S)} \int_{S}>\left|U_{\alpha}\left(x, \omega_{N}^{0}\right)\right| d \sigma(x)
$$

(The reader should compare Theorem A with Theorem 1 in [4].)

Part (d) of the assertion describes an exceptional case: if $\alpha$ is a positive even integer, the function $U_{\alpha}\left(x, \omega_{N}\right)$ is a trigonometric polynomial in the spherical coordinates of $S^{d-1}$. Note that the logarithmic case $\alpha=0$ for dimension $d=3$ has already been treated in [3]. In [4] we also considered, for a given set $\omega_{N}=\left\{x_{1}, x_{2}, \ldots, x_{N}\right\}$ of points of $S^{d-1}$, distance functionals $E_{\alpha}\left(\omega_{N}\right)$ defined by

$$
\begin{aligned}
& E_{\alpha}\left(\omega_{N}\right)=\sum_{j=1}^{N} \sum_{k=1}^{N}\left(\left|x_{j}-x_{k}\right|^{\alpha}-m(\alpha, d)\right) \quad \text { for } 0<\alpha<2, \\
& E_{0}\left(\omega_{N}\right)=\sum_{j \neq k} \sum_{j}\left(\log \left|x_{j}-x_{k}\right|-m(0, d)\right),
\end{aligned}
$$

and

$$
E_{\alpha}\left(\omega_{N}\right)=\sum_{j \neq k}\left(\left|x_{j}\right|^{\alpha}-m(\alpha, d)\right) \quad \text { for } 1-d<\alpha<0 .
$$

For $0<\alpha<2$ and $N \geq 2$, the sum $E_{\alpha}\left(\omega_{N}\right)$ is known to be negative (see Theorem 2 in [4]).

An application of Theorem A immediately yields 
THEOREM B. For any $\alpha$ with $0<\alpha<2$ and some positive constant $c(\alpha, d)$ there exists, for each $N \geq 2$, an $N$-tuple $\omega_{N}^{0}$ of points on $S^{d-1}$ (depending on $\alpha$ ) such that the following inequality holds:

$$
E_{\alpha}\left(\omega_{N}^{0}\right) \geq-c_{1}(\alpha, d) \cdot N^{1-\alpha /(d-1)} .
$$

Here $c_{1}(\alpha, d)$ is a positive constant, independent of $N$.

Theorem B shows that the inequality proved in [4] (Theorem 2(a)) is best possible, apart from the value of $c_{1}(\alpha, d)$. We remark that the special case $\alpha=1$ has already been proved by K. B. Stolarsky [2].

The situation for the sums $E_{\alpha}\left(\omega_{N}\right)$ in the unbounded case $1-d<$ $\alpha \leq 0$ is more complicated. The bounds obtained in [4] are thought to be best possible only for parameters $\alpha$ satisfying $1-d<\alpha \leq 3-d$. Unlike as in the preceding case, Theorem A can no longer be used to derive the existence of "good" point sets $\omega_{N}^{0}$. Instead, we give a direct construction of such point sets, but only for spheres in threedimensional space. We have the following proposition:

Theorem C. Let $d=3$. For any $\alpha$ with $-2<\alpha<0$ and some positive constant $c_{1}(\alpha)$ there exists, for each $N \geq 2$, an $N$-tuple $\omega_{N}^{0}$ of points on $S^{2}$ such that

$$
E_{\alpha}\left(\omega_{N}^{0}\right) \leq-c_{1}(\alpha) \cdot N^{1-\alpha / 2} .
$$

Similarly, for $\alpha=0$ and $N \geq 2$, there exists an $\omega_{N}^{0}$ such that

$$
E_{0}\left(\omega_{N}^{0}\right) \geq \frac{N}{2} \cdot \log +O(N) .
$$

Note that the logarithmic case has already been handled in the author's paper [3]. There the construction of the set $\omega_{N}^{0}$ is described completely, but the proof of relation (2), due to its highly computational nature, is only sketched. This unpleasant situation prevails even more in the case $-2<\alpha<0$, and so again we shall omit the computational details.

For a physical interpretation of results in the special case $\alpha=-1$, $d=3$, we refer to the author's paper [4].

2. Proof of Theorems A and B. The construction of "good" point sets $\omega_{N}^{0}$ for the proof of Theorems $\mathrm{A}$ and $\mathrm{B}$ depends on a result ("Main Lemma") on numerical integration with equal weights. As usual, the spherical coordinates on $S^{d-1}$ are denoted by $\theta_{1}, \theta_{2}, \ldots$, $\theta_{d-2}\left(0 \leq \theta_{\mu} \leq \pi\right)$ and $\phi(0 \leq \phi<2 \pi)$. Futhermore, we denote 
by $\Omega_{r}$ the set of trigonometric polynomials in the variables $\theta_{1}, \theta_{2}$, $\ldots, \theta_{d-2}$ and $\phi$, of degree not exceeding $r$, i.e. polynomials of the form

$$
\begin{aligned}
& p\left(\theta_{1}, \theta_{2}, \ldots, \theta_{d-2}, \phi\right) \\
&= \sum_{\left|j_{\mu}\right| \leq r,|k| \leq r} a\left(j_{1}, j_{2}, \ldots, j_{d-2}, k\right) \\
& \cdot \exp \left(i \cdot\left(\sum_{\mu=1}^{d-2} j_{\mu} \theta_{\mu}+k \cdot \phi\right)\right),
\end{aligned}
$$

where $j_{\mu}(\mu=1,2, \ldots, d-2)$ and $k$ are integers, and the $a\left(j_{1}, \ldots, j_{d-2}, k\right)$ are arbitrary complex coefficients.

MaIN Lemma. For all $d \geq 3$ and all $r \in N$ there exists an $n_{0}=$ $n_{0}(r, d)$ such that, for all domains $D \subseteq S^{d-1}$ of the form

$$
D=\left\{\left(\theta_{1}, \ldots, \theta_{d-2}, \phi\right): \beta_{1 \mu} \leq \theta_{\mu} \leq \beta_{2 \mu}, \quad \gamma_{1} \leq \phi \leq \gamma_{2}\right\},
$$

the following is true:

For each $(d-1)$-tuple of integers $\left(m_{1}, m_{2}, \ldots, m_{d-2}, n\right)$ satisfying $m_{j} \geq n_{0}(j=1, \ldots, d-2)$ and $n \geq n_{0}$, there is a set $P$ of $n$. $\prod_{j=1}^{d-2} m_{j}$ points $\left(\theta_{\mu_{1}}, \theta_{\mu_{2}}, \ldots, \theta_{\mu_{d-2}}, \phi_{\nu}\right) \quad\left(1 \leq \mu_{j} \leq m_{j}, 1 \leq \nu<n\right)$ on $D$ with the property that

$$
\frac{\sigma(D)}{\operatorname{card} P} \sum_{u \in P} p(u)=\int_{D} p(u) d \sigma(u)
$$

for each trigonometric polynomial $p(u)=p\left(\theta_{1}, \theta_{2}, \ldots, \theta_{d-2}, \phi\right) \in$ $\Omega_{R}$.

Let us make a few remarks.

(1) The mere existence of the number $n_{0}(r, d)$ for a given fixed domain $D$ follows from a general result of P.D. Seymour and T. Zaslavsky [1]. However, we need independence of the bound $n_{0}(r, d)$ from the special choice of the domain $D$. As the proofs given in [1] are not constructive, the results of these two authors cannot be used for our purpose.

(2) We may consider formula (3) as a quadrature formula with equal weights for the system of functions $\Omega_{r}$. A classical negative result for ordinary polynomials on an interval (due to $\mathrm{S}$. N. Bernstein) shows that we may not expect the bound $n_{0}(r, d)$ to be of an order as small as $r^{d-1}$. 
(3) In order not to interrupt the line of the proof of Theorems A and $\mathrm{B}$, we shall postpone the proof of the Main Lemma to the end of the paper.

The proof of Theorem A splits into several cases according to the value of the parameter $\alpha$.

The case $0<\alpha<2$. Let $\alpha$ be fixed, and let $n_{0}=n_{0} \quad(r=d, d)$ be the number the existence of which is guaranteed by the Main Lemma. Let $N$ be sufficiently large, $N=k \cdot n_{0}^{d-1}+l$, where $0 \leq l<n_{0}^{d-1}$ and $k=\left[N / n_{0}^{d-1}\right]$. By cutting the coordinate intervals $0 \leq \theta_{\mu} \leq \pi$ $(\mu=1,2, \ldots, d-2)$ and $0 \leq \phi<2 \pi$ into pieces appropriately, it is not difficult to see that we may divide the surface $S^{d-1}$ into subdomains $D_{1}, D_{2}, \ldots, D_{t}, t=t(N)$, which are "rectangles" in the system of spherical coordinates, and which possess the following basic properties:

(a) We have $\sigma\left(D_{\tau}\right)=N^{-1} \cdot n_{0}^{d-1} \cdot \sigma(S)$ for $\tau=1,2, \ldots, t-1$, and for $\tau=t$ if $l=0$, and $\sigma\left(D_{t}\right)=N^{-1} \cdot l \cdot\left(1+n_{0}^{d-1}\right)^{d-1} \cdot \sigma(S)$ if $0<l<n_{0}^{d-1}$.

(b) Denoting by $\left|D_{\tau}\right|=\sup _{x, y \in D_{\tau}}|x-y|$ the diameter of $D_{\tau}$, we have

$$
\left|D_{\tau}\right| \leq c_{2} \cdot N^{-1 /(d-1)} \quad(\tau=1,2, \ldots, t),
$$

where $c_{2}$ is a positive constant depending on the dimension $d$ only.

We apply the Main Lemma to each of the domains $D_{\tau}$. We choose $m_{1}=m_{2}=\cdots=m_{d-2}=n=n_{0}$ for the domains $D_{1}, \ldots, D_{t-1}$, and for $D_{t}$ if $l=0$, and $m_{1}=m_{2}=\cdots=m_{d-2}=\left(1+n_{0}^{d+1}\right)$, $n=l \cdot 1+n_{0}^{d-1}$ for $D_{t}$ if $l>0$. The set of interpolation points, distributed on each $D_{\tau}$ according to the Main Lemma, will be denoted by $P_{\tau}$, where card $P_{\tau}=n \cdot \prod_{j=1}^{d-2} m_{j}$, with $m_{j}, n$ as defined above.

Let $z_{\tau}=\left(\frac{1}{2}\left(\beta_{1}+\beta_{1}^{\prime}\right), \ldots, \frac{1}{2}\left(\beta_{d-2}+\beta_{d-2}^{\prime}\right), \frac{1}{2}\left(\gamma+\gamma^{\prime}\right)\right)$ be the "midpoint" of the domain $D_{\tau}=\left\{\beta_{\mu} \leq \theta_{\mu} \leq \beta_{\mu}^{\prime}, \gamma \leq \phi \leq \gamma^{\prime}\right\}$. (This choice of $z_{\tau}$ on $D_{\tau}$ is rather arbitrary.)

Fix $x \in S^{d-1}$, and denote by $\bar{D}_{\tau}$ the convex hull of $D_{\tau}$ in $d$ dimensional space $E^{d}$. By relation (4), there are at most $O(1)$ domains $D_{\tau}$ for which the inequality

$$
|x-y| \leq c_{2} \cdot N^{-1 /(d-1)}
$$

holds for some point $y \in \bar{D}_{\tau}$. (For simiplicity, we use the same constant $c_{2}$ in (4) and (5).) On each of these $O(1)$ domains, the 
following inequality is true:

(6)

$$
\begin{aligned}
\mid \frac{N}{\sigma(S)} & \int_{D_{\tau}}|x-y|^{\alpha} d \sigma(y)-\sum_{u \in P_{\tau}}|x-u|^{\alpha} \mid \\
\leq & \frac{N}{\sigma(S)} \cdot \sigma\left(D_{\tau}\right) \cdot\left(2 c_{2} \cdot N^{-1 /(d-1)}\right)^{\alpha} \\
& +\sum_{u \in P_{\tau}}\left(2 c_{2} \cdot N^{-1 /(d-1)}\right)^{\alpha} \ll N^{-\alpha /(d-1)} .
\end{aligned}
$$

Let $M_{q}(q=1,2, \ldots)$ be the class of domains $D_{\tau}$ such that

$$
q \cdot c_{2} \cdot N^{-1 /(d-1)} \leq \min _{y \in \bar{D}_{\tau}}|x-y|<(q+1) \cdot c_{2} \cdot N^{-1 /(d-1)} .
$$

By (4), there are at most $\ll q^{d-2}$ domains $D_{\tau}$ in $M_{q}$. On each $D_{\tau}$ of $M_{q}$, consider the Taylor expansion

(7) $|x-y|^{\alpha}=\left|x-z_{\tau}\right|^{\alpha}$

$$
\begin{aligned}
& +\sum_{m=1}^{d} \frac{1}{m !}\left(\left(y-z_{\tau}\right) \operatorname{grad}_{w}\right)^{m}|x-w|_{w=z_{\tau}}^{\alpha}+R(x, y) \\
= & T_{d}(x, y)+R(x, y),
\end{aligned}
$$

where

$$
\begin{aligned}
R(x, y)=\frac{1}{(d+1) !}\left(\left(y-z_{\tau}\right) \operatorname{grad}_{w}\right)^{d+1}|x-w|_{w=z_{\tau}+\delta\left(y-z_{\tau}\right)}^{\alpha}, & 0<\delta<1 .
\end{aligned}
$$

The remainder term can be estimated as

$$
\begin{aligned}
|R(x, y)| & \ll N^{-(d+1) /(d-1)} \cdot\left(q \cdot N^{-1 /(d-1)}\right)^{\alpha-d-1} \\
& \ll q^{\alpha-d-1} \cdot N^{-\alpha /(d-1)} .
\end{aligned}
$$

The main term $T_{d}(x, y)$ is a polynomial in the cartesian coordinates of $y$ of degree $\leq d$ which, after introducing spherical coordinates, becomes a trigonometric polynomial in $\theta_{1}, \ldots, \theta_{d-2}, \phi$ of the class $\Omega_{d}$, again of degree $\leq d$. By our choice of the point set $P_{\tau}$, we have

$$
\frac{N}{\sigma(S)} \int_{D_{\tau}} T_{d}(x, y) d \sigma(y)=\sum_{u \in P_{\tau}} T_{d}(x, u)
$$

Hence, for each $D_{\tau}$ in $M_{q}$, noting (8), we have the inequality

$$
\left|\frac{N}{\sigma(S)} \int_{D_{\tau}}\right| x-\left.y\right|^{\alpha} d \sigma(y)-\sum_{u \in P_{\tau}}|x-y|^{\alpha} \mid \ll q^{\alpha-d-1} \cdot N^{-\alpha /(d-1)} \text {. }
$$


Here, as in the preceding inequalities, the constants implicit in the Vinogradov symbols $\ll$ may depend on $\alpha$ and $d$, but are independent of $q, \tau, x$, and $N$. Summing over all classes $M_{q}$ and noting (6), we finally obtain:

$$
\begin{aligned}
& \left|\frac{N}{\sigma(S)} \int_{S}\right| x-\left.y\right|^{\alpha} d \sigma(y)-\sum_{\tau=1}^{t} \sum_{u \in P_{\tau}}|x-u|^{\alpha} \mid \\
& \ll N^{-\alpha /(d-1)}+\sum_{q=1}^{\infty} q^{d-2} \cdot q^{\alpha-d-1} \cdot N^{-\alpha /(d-1)} \ll N^{-\alpha /(d-1)} .
\end{aligned}
$$

This proves Theorem $\mathrm{A}$ in the case $0<\alpha<2$, and Theorem $\mathrm{B}$.

The case $2 \leq \alpha<\infty$. In the cases $2<\alpha<4,4<\alpha<6, \ldots$, we proceed as before, choosing successively $r=d+2, d+4, \ldots$, and approximating $|x-y|^{\alpha}$ by a Taylor polynomial of degree $\leq r$. In the case $\alpha=2 h \quad(h=1,2, \ldots)$, note that $|x-y|^{\alpha}$ is a trigonometric polynomial of degree $2 h$ in the variables $\theta_{1}, \ldots, \theta_{d-2}, \phi$. Choosing $D=S$ and $r=2 h$ in the Main Lemma, the assertion follows.

The case $1-d<\alpha \leq 0$. We proceed as in the case $0<\alpha<2$, choosing $r=d$ in the Main Lemma. The only difference in the argument concerns the derivation of the estimate (6), which has to be replaced in the following way: For fixed $x$ on $S$, consider again those domains $D_{\tau}$ for which $|x-y| \leq c_{2} \cdot N^{-1 /(d-1)}$ holds for some point $y$ in the convex hull of $D_{\tau}$. Then the following one-sided estimate is true for $\alpha<0$ :

$$
\sum_{u \in P_{\tau}}|x-u|^{\alpha}-\frac{N}{\sigma(S)} \int_{D_{\tau}}|x-y|^{\alpha} d \sigma(y) \geq-c_{3}(\alpha, d) \cdot N^{-\alpha /(d-1)}
$$

In order to prove (9), we simply omit the sum and estimate the integral from above, using relation (4). In the logarithmic case $\alpha=0$, the corresponding inequality is

$$
\sum_{u \in P_{\tau}} \log |x-u|-\frac{N}{\sigma(S)} \int_{D_{\tau}} \log |x-y| d \sigma(y) \leq-c_{4}(d) .
$$

Here the sum cancels the logarithmic part of the integral, leaving a remainder which is bounded from above.

From (8), (9), and (10) the assertion follows. This finishes our proof of Theorem A. 
3. Outline of a proof of Theorem $C$. The method of constructing "good" point sets $\omega_{N}^{0}$ in the case $d=3,-2<\alpha<0$, is of a similar type as the one given in $\S 2$. The verification of the inequality in Theorem $\mathrm{C}$, however, requires careful direct estimation.

We begin by describing the construction.

Let $\alpha$ and $N \geq 2$ be fixed. Put $N=[\sqrt{N \cdot b}]$, where $B$ is a positive constant to be determined later. Denoting the spherical coordinates on $S^{2}$ as usual by $\theta \quad(0 \leq \theta \leq \pi)$ and $\phi \quad(0 \leq \phi<2 \pi)$, we define angles $\theta_{0}, \ldots, \theta_{M}$ by the conditions

$$
0=\theta_{0}<\theta_{1}<\cdots<\theta_{M}=\pi
$$

such that $N_{\mu}:=\frac{N}{2}\left(\cos \theta_{\mu-1}-\cos \theta_{\mu}\right)(\mu=1,2, \ldots, M)$ are positive integers, and such that

$$
K_{1} / \sqrt{b \cdot N} \leq \theta_{\mu}-\theta_{\mu-1} \leq K_{2} \sqrt{b \cdot N}
$$

holds for $b<\mu<M-b$ and certain numerical constants $0<K_{1}<$ $K_{2}$. Each zone $D_{\mu}:=\left\{(\theta, \phi): \theta_{\mu-1} \leq \theta \leq \theta_{\mu}\right\}$ is divided into $N_{\mu}$ subdomains $D_{\mu j}$, where

$$
\begin{aligned}
& D_{\mu j}=\left\{(\theta, \phi): \theta_{\mu-1} \leq \theta \leq \theta_{\mu},\right. 2 \pi \cdot N_{\mu}^{-1}\left(j-\frac{1}{2}\right) \\
&\left.\leq \phi \leq 2 \pi \cdot N_{\mu}^{-1}\left(j+\frac{1}{2}\right)\right\} \\
&\left(\mu=1, \ldots, M ; j=0, \ldots, N_{\mu}-1\right) .
\end{aligned}
$$

On each $D_{\mu j}$, we choose a point $x_{\mu j}=\left(\xi_{\mu}, \phi_{\mu j}\right)$, where $\phi_{\mu j}=$ $2 \pi j / N_{\mu}$ and $\cos \xi_{\mu}=\frac{1}{2}\left(\cos \theta_{\mu-1}+\cos \theta_{\mu}\right)$. Let $\omega_{N}^{0}=\left\{x_{\mu j}\right\}$. By a heuristic argument we will try to explain why the set $\omega_{N}^{0}$ can be expected to satisfy inequality (1).

For fixed $x_{\mu j}$, the term $\left|x_{\mu j}-x_{\nu k}\right|^{\alpha}$ is roughly equal to the integral

$$
\frac{N}{4 \pi} \int D_{\nu k}\left|x_{\mu j}-y\right|^{\alpha} d \sigma(y)
$$

hence the whole sum

$$
\sum_{(\mu, j) \neq(\nu, k)}\left(\left|x_{\mu j}-x_{\nu k}\right|^{\alpha}-m(\alpha, 3)\right)
$$

corresponds to the sum of integrals

$$
-\sum_{(\mu, j)} \frac{N}{4 \pi} \int_{D_{\mu, j}}\left(\left|x_{\mu j}-y\right|^{\alpha}-m(\alpha, 3)\right) d \sigma(y) \text {. }
$$


This latter sum (12) is easily seen to be

$$
\leq-c_{5} \cdot b^{-1-(\alpha / 2)} \cdot N^{1-(\alpha / 2)} \text {. }
$$

What remains to be shown is the fact that the error which we commit when replacing (11) by (12), is of smaller order than the bound (13). This turns out to be true if we choose $b$ large enough, and if the numbers $N_{\mu}$ satisfy some additional condition of arithmetical nature. The proof, however, is too laborious to be presented here.

4. On quadrature formulas with equal weights. The Main Lemma will be derived from the following theorem which may be of independent interest in itself.

THEOREM. Let $w(x) \geq 0$ be an integral weight function on the interval $[-1,1]$, satisfying the relations $\int_{-1}^{1} w(x) d x=1$ and

$$
L_{1} \geq w(x) \geq L_{2} \cdot(1-|x|)^{\beta},
$$

with constants $L_{1}>0, L_{2}>0$, and $\beta>0$. Let $\Phi=\left\{\phi_{1}, \ldots, \phi_{s}\right\}$ be a system of three times continuously differentiable functions on $[-1,1]$, with the additional property that the derivatives $\phi_{1}^{\prime}, \phi_{2}^{\prime}, \ldots, \phi_{s}^{\prime}$ form an orthonormal system with respect to the weight function $w(x)$. Let

$$
K_{1}=\max _{[-1,1]} \max _{\mu=1, \ldots, s}\left(\left|\phi_{\mu}^{\prime}\right|,\left|\phi_{\mu}^{\prime \prime}\right|,\left|\phi_{\mu}^{\prime \prime \prime}\right|\right) \text {. }
$$

Then there exists a number $n_{0}$, depending only on $L_{1}, L_{2}, K_{1}, \beta$, and $s$, such that for each $n \geq n_{0}$, there exist points $t_{1}, t_{2}, \ldots, t_{n}$ with $-1<t_{1}<t_{2}<\cdots<t_{n}<1$ and

$$
\frac{1}{n} \sum_{j=1}^{n} \phi_{\mu}\left(t_{j}\right)=\int_{-1}^{1} \phi_{\mu}(x) w(x) d x
$$

for all $\phi_{\mu} \in \Phi$ simultaneously.

Proof. 1. In the sequel we will have to deal with the functions $\phi_{\mu}^{\prime}$, $\phi_{\mu}^{\prime} \phi_{\nu}^{\prime} \quad(\mu, \nu=1,2, \ldots, s)$, and their derivatives up to the second order. By our assumption (15), all these functions are bounded in absolute value by

$$
K:=\max \left(K_{1}, 4 K_{1}^{2}\right) .
$$

For the construction of the point set $\left\{t_{j}\right\}$, we use Newton's method. We begin by defining intervals $I_{j}=\left[x_{j-1}, x_{j}\right]$ by the relation

$$
\int_{-1}^{x_{j}} w(x) d x=\frac{j}{n} \quad(j=0,1, \ldots, n) .
$$


By assumption (14), we have $\left(\left|I_{j}\right|=\right.$ length of $\left.I_{j}\right)$

$$
\left(L_{1} \cdot n\right)^{-1} \leq\left|I_{j}\right| \leq 2 \cdot\left(L_{2} \cdot \gamma \cdot n\right)^{-\gamma},
$$

where we write $\gamma:=1 /(\beta+1)$ for the sake of brevity.

In the interior of each interval $I_{j}$, choose the (uniquely determined) point $\xi_{j}$ with the property that

$$
\int_{I_{j}}\left(x-\xi_{j}\right) w(x) d x=0 .
$$

By the assumption $w(x) \leq L_{1}$ in (14), the following inequality holds:

$$
\min \left(x_{j}-\xi_{j}, \xi_{j}-x_{j-1}\right) \geq\left(2 L_{1} \cdot n\right)^{-1} \text {. }
$$

We use the point set $\left\{\xi_{j}\right\}$ as the starting point of a Newton iteration process. By changing the values of $\xi_{j}$ successively, we obtain a sequence of $n$-point sets on $[-1,1]$, converging to a set $-1<t_{1}<$ $\cdots<t_{n}<1$ with the desired property (16), provided that the number $n$ is chosen large enough. We remark here that if not otherwise stated, all the constants that appear in the following parts of the proof are assumed to depend on $L_{1}, L_{2}, K_{1}, \beta, s$, but not on $n$.

2 . Let $f$ be any function on $[-1,1]$, twice continuously differentiable and satisfying the relation

$$
\max _{[-1,1]}\left(\left|f^{\prime}(x)\right|,\left|f^{\prime \prime}(x)\right|\right) \leq K \text {. }
$$

By Taylor's theorem, using (18), we have the following basic estimate:

$$
\begin{gathered}
\left|n \int_{I_{j}} f(x) w(x) d x-f\left(\xi_{j}\right)\right|=n\left|\int_{I_{j}}\left(f(x)-f\left(\xi_{j}\right)\right) w(x) d x\right| \\
=\frac{n}{2}\left|\int_{I_{j}}\left(x-\xi_{j}\right)^{2} f^{\prime \prime}(\xi(x)) w(x) d x\right| \leq \frac{K}{2} \cdot\left|I_{j}\right|^{2} .
\end{gathered}
$$

Summing over all intervals $I_{j}$, and noting (17), we obtain:

$$
\left|\sum_{j=1}^{n} f\left(\xi_{j}\right)-n \int_{-1}^{1} f(x) w(x) d x\right| \leq \frac{K}{2} \sum_{j=1}^{n}\left|I_{j}\right|^{2} \leq c_{1} \cdot n^{-\gamma} .
$$

Now assume that $-1<\eta_{1}<\cdots<\eta_{n}<1$ is a new set of points, satisfying $\left|\xi_{j}-\eta_{j}\right| \leq \delta$ for $j=1,2, \ldots, n$, and some real $\delta>0$. By (20) and (21) we have the estimate

$$
\left|\sum_{j=1}^{n} f\left(\eta_{j}\right)-n \int_{-1}^{1} f(x) w(x) d x\right| \leq c-1 \cdot n^{-\gamma}+\delta \cdot n \cdot K .
$$


3. Without loss of generality we may assume that $\int_{-1}^{1} \phi_{\mu}(x) w(x) d x$ $=0$ holds for all $\phi_{\mu}$ in $\Phi$. Suppose that after the $r$ th step of the iteration procedure we arrive at a point set $-1<\eta_{1}<\cdots<\eta_{n}<1$ $\left(r=0\right.$ describes the initial situation $\left.\eta_{j}=\xi_{j}\right)$ with the following two properties:

$$
\left|\sum_{j=1}^{n} f\left(\eta_{j}\right)-n \int_{-1}^{1} f(x) w(x) d x\right| \leq C_{r} \cdot n^{-\gamma}
$$

for each $f$ satisfying relation (20), and

$$
\sum_{j=1}^{n} \phi_{\mu}\left(\eta_{j}\right)=\rho_{\mu} \quad(\mu=1, \ldots, s),
$$

where $\left|\rho_{\mu}\right| \leq \sigma_{r}$ for all values of $\mu$, and $C_{r}, \sigma_{r}$ are positive constants which may depend on $r$.

Put $\eta_{j}^{\prime}=\eta_{j}-h_{j}$. Replacing $\eta_{j}$ by $\eta_{j}^{\prime}$ in (24), and linearizing, we obtain the following linear system of equations for the corrections $h_{j}$ :

$$
\sum_{j=1}^{n} h_{j} \phi_{\mu}^{\prime}\left(\eta_{j}\right)=\rho_{\mu} \quad(\mu=1, \ldots, s) .
$$

We are looking for a solution vector $\left(h_{1}, h_{2}, \ldots, h_{n}\right)$ of $(25)$ with all the $h_{j}$ being small. Here we make essential use of the orthogonormality of the derivatives $\phi_{\mu}^{\prime}$ with respect to $w(x)$. We interpret the system (25) as a set of hyperplanes in Euclidean $n$-space. By (23), we obtain the following estimates for the scalar products between their normal vectors $\left(\phi_{\mu}^{\prime}\left(\eta_{1}\right), \ldots, \phi_{\mu}^{\prime}\left(\eta_{n}\right)\right)$ :

$$
\left|\sum_{j=1}^{n} \phi_{\mu}^{\prime}\left(\eta_{j}\right) \phi_{\nu}^{\prime}\left(\eta_{j}\right)\right|=:\left|A_{\mu \nu}\right| \leq C_{r} \cdot n^{-\gamma} \quad(\mu \neq \nu),
$$

and

$$
\sum_{j=1}^{n} \phi_{\nu}^{\prime 2}\left(\eta_{j}\right)=: A_{\mu \mu} \geq n-C_{r} \cdot n^{-\gamma} .
$$

An application of Lagrange's method (with multipliers $\lambda_{\nu}$ ) to the expression

$$
\sum_{j=1}^{n} h_{j}^{2}-\sum_{\nu=1}^{s} \lambda_{\nu}\left(\sum_{j=1}^{n} h_{j} \phi_{\nu}^{\prime}\left(\eta_{j}\right)-\rho_{\nu}\right)
$$


leads to a minimal solution of (25). For the multipliers $\lambda_{\nu}$ we obtain the following linear system of equations:

$$
2 \cdot h_{j}=\sum_{\nu=1}^{s} \lambda_{\nu} \cdot \phi_{\nu}^{\prime}\left(\eta_{j}\right) \quad(j=1,2, \ldots, n) .
$$

Multiplying both sides of (27) by $\phi_{\mu}^{\prime}\left(\eta_{j}\right)$ and summing over $j$, we obtain, using (26), a new system of equations:

$$
2 \cdot \pi_{\mu}=\sum_{\nu=1}^{s} A_{\mu \nu} \lambda_{\nu} \quad(\mu=1,2, \ldots, s) .
$$

The matrix of the system (28) is approximately diagonal in view of (26). We have the decomposition

$$
\left(A_{\mu \nu}\right)=\left(\begin{array}{llll}
A_{11} & & & 0 \\
& A_{22} & & \\
& & \ddots & \\
0 & & & A_{s s}
\end{array}\right) \cdot\left(\begin{array}{llll}
1 & & & * \\
& 1 & & \\
& & \ddots & \\
* & & & 1
\end{array}\right)=D \cdot(I+B),
$$

$(I=$ identity matrix $)$, where the entries of $B$ are

$$
\leq C_{r} \cdot n^{-\gamma} /\left(n-C_{r} \cdot n^{-\gamma}\right)
$$

in absolute value. Let us calculate the inverse matrix $\left(A_{\mu \nu}\right)^{-1}$ :

$$
\left(A_{\mu \nu}\right)^{-1}=\left(I-B+B^{2}-+\cdots\right) \cdot D^{-1}=\left(I+B_{1}\right) \cdot D^{-1},
$$

where the entries of $B_{1}$ are $\leq C_{r} \cdot n^{-\gamma} /\left(n-(s+1) C_{r} \cdot n^{-\gamma}\right)$ in absolute value. Hence the entries of the inverse $\left(A_{\mu \nu}\right)^{-1}$ are in absolute value

$$
\leq\left(n^{1+\gamma}-s \cdot C_{r}\right) \cdot\left(n^{1+\gamma}-(s+1) C_{r}\right)^{-1} \cdot\left(n-C_{r} \cdot n^{-\gamma}\right)^{-1}
$$

in the main diagonal, and by

$$
\leq C_{r} \cdot n^{\gamma} \cdot\left(n^{1+\gamma}-(s+1) C_{r}\right)^{-1} \cdot\left(n^{1+\gamma}-C_{r}\right)^{-1}
$$

elsewhere. Inserting these estimates into (28) and (27), we obtain the following inequalities:

$$
\left|\lambda_{\nu}\right| \leq 2 \sigma_{r} \cdot n^{\gamma} \cdot\left(n^{1+\gamma}-(s+1) C_{r}\right)^{-1} \quad(\nu=1, \ldots, s)
$$

and

$$
\left|h_{j}\right| \leq s K \sigma_{r} \cdot n^{\gamma} \cdot\left(n^{1+\gamma}-(s+1) C_{r}\right)^{-1} \quad(j=1, \ldots, n) .
$$


If we replace $\eta_{j}^{\prime}=\eta_{j}-h_{j}$ in (24), the new error terms $\rho_{\nu}^{\prime}$ are bounded in absolute value by

$$
\begin{aligned}
\sigma_{r+1} & \leq \sum_{j=1}^{n} \frac{1}{2} h_{j}^{2} \cdot \max _{\nu} \max _{x \in I_{j}}\left|\phi_{\nu}^{\prime \prime}(x)\right| \\
& \leq \frac{n}{2} K^{3} s^{2} \sigma_{r}^{2}\left(n-(s+1) C_{r} n^{-\gamma}\right)^{-2} .
\end{aligned}
$$

By (29) and (22) the new constant $C_{r+1}$ in (23) can be chosen as small as

$$
C_{r+1} \leq C_{r}+\sigma_{r} \cdot s K^{2} n^{1+\gamma}\left(n-(s+1) C_{r} n^{-\gamma}\right)^{-1} .
$$

Keeping in mind that $\sigma_{0} \leq C_{0} n^{-\gamma}$ by (21), it is not difficult to prove by induction from (30) and (31) that if we choose the number $n$ of interpolation points large enough, the following inequalities are true:

$$
\sigma_{r} \leq\left(C_{0} n^{-\gamma}\right)^{2^{r}}, \quad C_{r+1}-C_{r} \leq c_{2} \cdot 2^{-r-1} \cdot C_{0} \quad \text { and } \quad C_{r} \leq 2 c_{2} \cdot C_{0} \text {. }
$$

Moreover, it follows from the second half of (29) that the total displacement of the initial points $\xi_{j}$ does not exceed

$$
c_{3} \cdot n^{-1} \cdot \sum_{r=0}^{\infty} \sigma_{r} \leq c_{4} \cdot n^{-1-\gamma} .
$$

Hence, in view of (19), all the limit points $t_{j}$ of the sequences $\xi_{j}, \ldots$, $\eta_{j}, \eta_{j}^{\prime}, \ldots$ are contained in the interval $(-1,1)$. This finishes the proof of the theorem.

In order to derive the Main Lemma from the preceding theorem we have to prove that the bound $K_{1}$ in condition (15) can be chosen such as to be independent of certain parameters connected with the choice of the domain $D$.

LEMMA. Let $w(x)$ be a weight function on $[-1,1]$, satisfying the conditions $\int_{-1}^{1} w(x) d x=1$ and $w(x) \geq L_{2}(1-|x|)^{\beta}$, where $\beta, L_{2}$ are positive constants. Let $\Psi=\left\{T_{0}(x), \ldots, T_{2 r}(x)\right\}$ be the system of functions on $[-1,1]$ defined by

$$
T_{2 j}(x)=\left(\frac{1-\cos \varepsilon x}{\varepsilon^{2} / 2}\right)^{j} \quad(j=0, \ldots, r)
$$

and

$$
T_{2 j+1}(x)=T_{2 j}(x) \cdot \frac{\sin \varepsilon x}{\varepsilon} \quad(j=0, \ldots, r-1) .
$$


Here $\varepsilon$ denotes a positive real parameter. If the functions $T_{k}$ are orthonormalized successively with respect to $w(x)$ by the Gram-Schmidt process, and if $\varepsilon<\varepsilon_{0}\left(L_{2}, r, \beta\right)$ holds, then the functions of the new system are bounded on $[-1,1]$ by a constant which depends on $r, L_{2}$ and $\beta$, but not on $\varepsilon$.

Proof. Let $\Psi_{1}=\left\{g_{0}=T_{0}, g_{1}, \ldots, g_{2 r}\right\}$ be the orthonormal system resulting from $\Psi$. Each $g_{j}$ has a unique representation of the form

$$
g_{j}(x)=b_{j 0} T_{0}(x)+\cdots+b_{j j} T_{j}(x) .
$$

Assume that for some $s, 0 \leq s<2 r$, the following inequality holds:

$$
\begin{aligned}
\left|b_{j k}\right| \leq K\left(s, L_{2}, \beta\right) & =K_{s} \\
& (j=0,1, \ldots, s ; k=0,1, \ldots, j) .
\end{aligned}
$$

Note that (33) is true for $s=0$ with $K_{0}=1$. We proceed by induction on $s$. We orthogonalize the function $T_{s+1}(x)$ with respect to $g_{0}, \ldots, g_{s}$ by setting

$$
f_{s+1}(x)=T_{s+1}(x)-\sum_{j=0}^{s}\left\langle g_{j}, T_{s+1}\right\rangle g_{j}(x)=\sum_{j=0}^{s+1} a_{j} T_{j}(x)
$$

and

$$
g_{s+1}(x)=f_{s+1}(x) /\left\|f_{s+1}\right\|_{2} .
$$

Here as usual we define $\langle f, g\rangle=\int_{-1}^{1} f(x) g(x) w(x) d x$ and $\|f\|_{2}^{2}=$ $\langle f, f\rangle$.

Note that $\left|T_{j}(x)\right| \leq 1$ on $[-1,1]$; hence $\left|\left\langle g_{j}, T_{s+1}\right\rangle\right| \leq 1$. From (32) and (33) it follows that

$$
\left|a_{j}\right| \leq(s+1) \cdot K_{s}
$$

for $j=0, \ldots, s+1$. All we have to prove is that $\left\|f_{s+1}\right\|_{2}$ is bounded from below. From the inequality

$$
\left|T_{j}(x)-x^{j}\right| \leq c_{1}(s) \cdot \varepsilon^{2},
$$

valid from $x \in[-1,1], j=0,1, \ldots, s+1$, and $\varepsilon<1$ it follows that $f_{s+1}(x)$ admits an approximation by a monic polynomial, i.e.

$$
f_{s+1}(x)=x^{s+1}+d_{s} x^{s}+\cdots+d_{0}+R(x)=p_{s}(x)+R(x),
$$

where $|R(x)|<\varepsilon^{2} \cdot c_{2}\left(s, L_{2}, \beta\right)$. Using expansion of $p_{s}(x)$ into 
Legendre polynomials, we easily obtain:

$$
\max _{[-1,1]}\left|f_{s+1}(x)\right| \geq c_{3}(s)-c_{2} \cdot \varepsilon^{2},
$$

where $c_{3}(s)>0$.

Furthermore, using $\left|f_{s+1}^{\prime}(x)\right| \leq \sum\left|a_{j}\right| \cdot\left|T_{j}^{\prime}(x)\right|<c_{4}\left(s, L_{2}, \beta\right)$, we find that $\left|f_{s+1}(x)\right|>\frac{1}{2} c_{3}(s)$ holds on an interval of length $\geq \delta\left(s, L_{2}, \beta\right)$ $>0$, provided that $\varepsilon$ is small enough. From the assumption $w(x) \geq$ $L_{2}(1-|x|)^{\beta}$ we obtain the estimate $\left\|f_{s+1}\right\|_{2} \geq c_{5}\left(s, L_{2}, \beta\right)>0$, which proves the assertion in view of the relations (34) and (35).

Corollary. As the derivatives $T_{j}^{\prime}(x)$ and $T_{j}^{\prime \prime}(x)(j=0, \ldots, 2 r)$ are bounded on $[-1,1]$, uniformly in $\varepsilon>\varepsilon_{0}$, it follows from (32) that the assertion of the lemma is also true for the derivatives $g_{j}^{\prime}$ and $g_{j}^{\prime \prime}$.

The proof of the Main Lemma is now completed as follows.

Let $D=\left\{\theta_{1 \mu} \leq \theta_{\mu} \leq \theta_{2 \mu}, \phi_{1} \leq \phi \leq \phi_{2}\right\} \subset S$ be the given domain. First we note that it is sufficient to prove the Main Lemma for domains $D$ for which the differences $\theta_{2 \mu}-\theta_{1 \mu}(\mu=1, \ldots, d-2)$ and $\phi_{2}-\phi_{1}$ are sufficiently small. In order to obtain the assertion for domains of arbitrary size, we only have to stick together a bounded number of suitable "small" $D$ 's. Secondly we note that it is sufficient to prove the existence of the bound $n_{0}(r)$ for each coordinate separately. Without restriction, we choose the coordinate $\theta_{1}$, the proof for the other coordinates being essentially the same. We are hence given the interval of integration $\theta_{11} \leq \theta \leq \theta_{21}$, the weight function $\sin ^{d-2} \theta_{1}$, and the system of functions $\Omega_{r}\left(\theta_{1}\right)=$ $\left\{1, \cos \theta_{1}, \ldots, \cos r \theta_{1}, \sin \theta_{1}, \ldots, \sin r \theta_{1}\right\}$. By a suitable linear transformation, replacing the variable $\theta_{1}$ by $x$, we obtain the interval $-1 \leq x \leq 1$, the weight function

$$
w(x)=\sin ^{d-2} \varepsilon\left(x-x_{0}\right) / \int_{-1}^{1} \sin d^{d-2} \varepsilon\left(x-x_{0}\right) d x,
$$

where $\varepsilon=\frac{1}{2}\left(\theta_{21}-\theta_{11}\right)$ and $x_{0}=\left(\theta_{21}+\theta_{11}\right) /\left(\theta_{21}-\theta_{11}\right)$, and the system $\Omega_{r}^{\prime}=\{1, \cos \varepsilon x, \ldots, \sin r \varepsilon x\}$. We replace the system $\Omega_{r}^{\prime}$ by the equivalent system $\Omega_{r}^{\prime \prime}=\left\{1, G_{0}, G_{1}, \ldots, G_{2 r}\right\}$, where $G_{0}, \ldots, G_{2 r}$ are arbitrary primitives of the functions $g_{0}, g_{2}, \ldots, g_{2 r}$ defined in the proof of the lemma. By the lemma, the assumptions of the theorem are now satisfied with $\beta=d-2, L_{1}$ and $L_{2}$ depending on $d$ only, and $K_{1} \leq c\left(L_{2}, \beta, r\right)=c(r)$, as soon as $\varepsilon<\varepsilon_{0}\left(L_{2}, \beta, r\right)=$ $\varepsilon_{0}(r)$. This finishes the proof of the Main Lemma. 


\section{REFERENCES}

[1] P. D. Seymour and T. Zaslavsky, Averaging sets, Adv. in Math., 52 (1984), 213-240.

[2] K. B. Stolarsky, Sums of distances between points of a sphere II, Proc. Amer. Math. Soc., 41 (1973), 575-582.

[3] G. Wagner, On the product of distances to a point set on a sphere J. Australian Math. Soc., (Series A) 47 (1989), 466-482.

[4] _ On means of distances on the surface of a sphere (Lower bounds), Pacific J. Math., 144 (1990), 389-398.

Received February 27, 1991.

Address for correspondence and reprints:

BODO VOLKMANN

Mathematisches Institut A

UNIVERSITÄT STUTTGART

PFAFFENWALdRING 57

D 7000 Stuttgart 80, Germany 


\section{PACIFIC JOURNAL OF MATHEMATICS EDITORS}

\author{
V. S. VARADARAJAN \\ (Managing Editor) \\ University of California \\ Los Angeles, CA 90024-1555 \\ Herbert Clemens \\ University of Utah \\ Salt Lake City, UT 84112 \\ F. Michael Christ \\ University of California \\ Los Angeles, CA 90024-1555 \\ THOMAS ENRIGHT \\ University of California, San Diego \\ La Jolla, CA 92093
}

\author{
Nicholas ERcolani \\ University of Arizona \\ Tucson, AZ 85721 \\ R. FINN \\ Stanford University \\ Stanford, CA 94305 \\ VAughan F. R. JONES \\ University of California \\ Berkeley, CA 94720 \\ C. C. Moore \\ University of California \\ Berkeley, CA 94720 \\ MARTIN ScharlemanN \\ University of California \\ Santa Barbara, CA 93106 \\ Harold Stark \\ University of California, San Diego \\ La Jolla, CA 92093
}

STEVEN KeRCKHOFF

Stanford University

Stanford, CA 94305

\begin{tabular}{|c|c|c|c|c|}
\hline \multicolumn{5}{|c|}{ ASSOCIATE EDITORS } \\
\hline R. Arens & $\begin{array}{ll}\text { E. F. BECKENBACH } & \text { B. H } \\
(1906-1982) & \end{array}$ & NeUmanN & $\begin{array}{c}\text { F. WoLF } \\
(1904-1989)\end{array}$ & K. Yoshida \\
\hline \multicolumn{5}{|c|}{ SUPPORTING INSTITUTIONS } \\
\hline \multicolumn{2}{|c|}{ UNIVERSITY OF ARIZONA } & \multicolumn{3}{|c|}{ UNIVERSITY OF OREGON } \\
\hline \multicolumn{2}{|c|}{ UNIVERSITY OF BRITISH COLUMBIA } & \multicolumn{3}{|c|}{ UNIVERSITY OF SOUTHERN CALIFORNIA } \\
\hline \multicolumn{2}{|c|}{ CALIFORNIA INSTITUTE OF TECHNOLOGY } & \multicolumn{3}{|c|}{ STANFORD UNIVERSITY } \\
\hline \multicolumn{2}{|c|}{ UNIVERSITY OF CALIFORNIA } & \multicolumn{3}{|c|}{ UNIVERSITY OF HAWAII } \\
\hline \multicolumn{2}{|c|}{ MONTANA STATE UNIVERSITY } & \multicolumn{3}{|c|}{ UNIVERSITY OF TOKYO } \\
\hline \multicolumn{2}{|c|}{ UNIVERSITY OF NEVADA, RENO } & \multicolumn{3}{|c|}{ UNIVERSITY OF UTAH } \\
\hline & \multicolumn{3}{|c|}{ WASHINGTON STATE UNIVERSITY } \\
\hline \multicolumn{2}{|c|}{ OREGON STATE UNIVERSITY } & \multicolumn{3}{|c|}{ UNIVERSITY OF WASHINGTON } \\
\hline
\end{tabular}

The Supporting Institutions listed above contribute to the cost of publication of this Journal, but they are not owners or publishers and have no responsibility for its content or policies.

Mathematical papers intended for publication in the Pacific Journal of Mathematics should be in typed
form or offset-reproduced (not dittoed), double spaced with large margins. Please do not use built up fractions
in the text of the manuscript. However, you may use them in the displayed equations. Underline Greek letters
in red, German in green, and script in blue. The first paragraph must be capable of being used separately as
a synopsis of the entire paper. In particular it should contain no bibliographic references. Please propose a
heading for the odd numbered pages of less than 35 characters. Manuscripts, in triplicate, may be sent to any
one of the editors. Please classify according to the 1991 Mathematics Subject Classification scheme which
can be found in the December index volumes of Mathematical Reviews. Supply name and address of author
to whom proofs should be sent. All other communications should be addressed to the managing editor, or
Elaine Barth, University of California, Los Angeles, California $90024-1555-05$.
There are page-charges associated with articles appearing in the Pacific Journal of Mathematics. These
charges are expected to be paid by the author's University, Government Agency or Company. If the author or
authors do not have access to such Institutional support these charges are waived. Single authors will receive
50 free reprints; joint authors will receive a total of 100 free reprints. Additional copies may be obtained at
cost in multiples of 50 .

The Pacific Journal of Mathematics (ISSN 0030-8730) is published monthly except for July and August. Regular subscription rate: $\$ 190.00$ a year (10 issues). Special rate: $\$ 95.00$ a year to individual members of supporting institutions.

Subscriptions, orders for numbers issued in the last three calendar years, and changes of address should be sent to Pacific Journal of Mathematics, P.O. Box 969, Carmel Valley, CA 93924, U.S.A. Old back numbers obtainable from Kraus Periodicals Co., Route 100, Millwood, NY 10546.

The Pacific Journal of Mathematics at P.O. Box 969, Carmel Valley, CA 93924 (ISSN 0030-8730) is published monthly except for July and August. Second-class postage paid at Carmel Valley, California 93924, and additional mailing offices. Postmaster: send address changes to Pacific Journal of Mathematics, P.O. Box 969, Carmel Valley, CA 93924.

PUBLISHED BY PACIFIC JOURNAL OF MATHEMATICS, A NON-PROFIT CORPORATION Copyright (C) 1992 by Pacific Journal of Mathematics 


\section{Pacific Journal of Mathematics}

\section{Vol. 154, No. $2 \quad$ June, 1992}

Manuel (Rodriguez) de León, J. A. Oubiña, P. R. Rodrigues and

Modesto R. Salgado, Almost $s$-tangent manifolds of higher order . . . . 201

Martin Engman, New spectral characterization theorems for $S^{2} \ldots \ldots \ldots 215$

Yuval Zvi Flicker, The adjoint representation $L$-function for GL $(n) \ldots \ldots 231$

Enrique Alberto Gonzalez-Velasco and Lee Kenneth Jones, On the range

of an unbounded partly atomic vector-valued measure . ............ 245

Takayuki Hibi, Face number inequalities for matroid complexes and

Cohen-Macaulay types of Stanley-Reisner rings of distributive

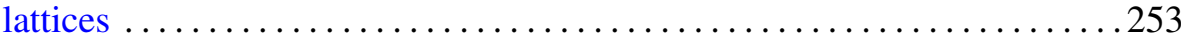

Hervé Jacquet and Stephen James Rallis, Kloosterman integrals for skew

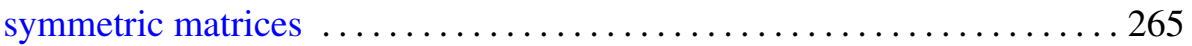

Shulim Kaliman, Two remarks on polynomials in two variables .........285

Kirk Lancaster, Qualitative behavior of solutions of elliptic free boundary problems ..........................................297

Feng Luo, Actions of finite groups on knot complements . ........... 317

James Joseph Madden and Charles Madison Stanton, One-dimensional

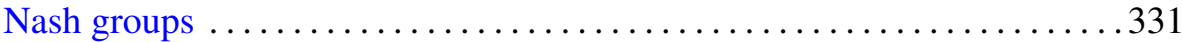

Christopher K. McCord, Estimating Nielsen numbers on

infrasolvmanifolds ......................................... 345

Gordan Savin, On the tensor product of theta representations of $\mathrm{GL}_{3}$ 369

Gerold Wagner, On means of distances on the surface of a sphere. II.

(Upper bounds) 